ICASSP'05, IEEE International Conference on Signal Processing,

Vol. II, Pages: 777-780, Philadelphia, PA, March 18-23, 2005.

\title{
ROBUST REORIENTATION OF 2D SHAPES USING THE ORIENTATION INDICATOR INDEX
}

\author{
Victor H. S. Ha* \\ Digital Media Solutions Lab \\ Samsung Information Systems America \\ Irvine, CA 92612 \\ \{v.ha@samsung.com\}
}

\author{
José M. F. Moura \\ Department of ECE \\ Carnegie Mellon University \\ Pittsburgh, PA 15213 \\ \{moura@ece.cmu.edu\}
}

\begin{abstract}
Shape reorientation is a critical step in many image processing and computer vision applications such as registration, detection, identification, and classification. Shape reorientation is a needed step to restore the correct orientation of a shape when its image is subject to an arbitrary rotation and reflection. In this paper, we present a robust method to determine the standard "normalized" orientation of twodimensional (2D) shapes in a blind manner, i.e., without any other information other than the given input shape. We introduce a set of orientation indicator indices (OII) that use low order central moments of the shape to monitor the orientational characteristics of the shape. Because these OII's use only low (up to third) order moments, they are robust to noise and errors. We show with examples how we bring consistently a given shape with an unknown arbitrary orientation to its standard normalized orientation using the OII.
\end{abstract}

\section{INTRODUCTION}

In image processing and computer vision applications, the shapes of objects are obtained and analyzed for registration, detection, identification, and classification. Many research efforts are directed to finding the correct orientation of the input shape prior to feeding it into such further processing.

We have developed the point-based reorientation algorithm (PRA) [1] and the variable-size window orientation indicator index $(\Delta$-OII) [2] that remove the orientational ambiguity from any shape distorted by an arbitrary orientation. The two algorithms are combined in a complete and efficient solution to the shape orientation problem, the PRA- $\Delta$-OII algorithm [2]. In this paper, we introduce the extended OII $(X$-OII). This new OII is resilient to error and noise, especially when there is an unexpectedly large amount of error or noise added to the shape.

${ }^{*}$ The first author performed the work while at Carnegie Mellon University. This work was partially supported by ONR Grant \#14-0-1-0593
In section 2, we briefly review some of the past research efforts on shape reorientation. In section 3 , we introduce three orientation indicator indices that we use to remove the orientation ambiguity in the shape: the orientation indicator index (OII), the variable-size window OII ( $\Delta$-OII), and the extended OII ( $X$-OII). We illustrate the good performance of these new measures of orientation with experimental examples. Section 4 summarizes the paper.

\section{SHAPE ORIENTATION}

There are an extensive number of published efforts to determine the orientation of shapes. A good set of existing techniques are reviewed in [3]; these techniques are shown to fail for some test shapes. The Shen-Ip symmetries detector [4] that survives these tests is concerned with the problem of detecting both the reflectional and rotational symmetry axes of a shape. The algorithm requires the computation of many high order generalized complex (GC) moments of the shapes.

In [1], we introduce the concept of intrinsic shape of an object as an invariant to the class of affine and permutation distortions of a shape, and summarize a blind algorithm to recover the intrinsic shape-BLAISER. The key step in BLAISER is the reorientation of the shape, which is solved with the point-based reorientation algorithm (PRA). The PRA provides a complete solution to the shape reorientation problem. The PRA is also an efficient algorithm whose complexity is $\mathcal{O}(N \log N)$ where $N$ is the number of feature points (pixels) in the given shape (binary template of the object). In practice, the locations of the feature points may be measured inaccurately due to the finite resolution of the input device and background noise. The PRA may be sensitive to such disturbances.

In the next section, we introduce a set of orientation indicator indices (OII) that are moment-based measures of the shape orientation. We use these OII's to develop a robust shape orientation algorithm. 


\section{ORIENTATION INDICATOR INDEX}

We now introduce the orientation indicator index (OII) that monitors the orientation of the shape. The OII uses a series of low order central moments of the input shape. Momentbased techniques are more robust to errors than point-based techniques such as the PRA. The OII is computed from up to third order central moments of the shape. We have chosen the third order moments because higher order moments are generally more sensitive to noise and other sources of error.

We start in subsection 3.1 with a definition of the OII using the third order central moments. Then, in subsection 3.2, we introduce the $\Delta$-OII, the variable-size window OII. Finally in subsection 3.3, we generalize the concept and introduce the extended orientation indicator index, $X$ OII. We illustrate with examples how these OII's apply to the shape orientation problem.

\subsection{OII}

We represent a 2D shape $\mathbf{X}$ as the collection of $\mathrm{x}$ - and $\mathrm{y}$ coordinates, $(x, y)$, falling inside the shape. The representation can be either continuous or discrete. We define the OII of shape $\mathbf{X}$ as

$$
\mathrm{OII}_{\mathbf{X}}=\sqrt{m_{30}^{2}+m_{03}^{2}}
$$

where $m_{p q}$ is the $(p+q)$ th order central moment of $\mathbf{X}$. If the shape $\mathbf{X}$ is rotated about its center of mass by an angle $\theta$, we define the OII of the rotated shape $\mathbf{X}_{\theta}$ by

$$
\mathrm{OII}_{\mathbf{X}_{\theta}}=\sqrt{\bar{m}_{30}^{2}+\bar{m}_{03}^{2}}
$$

where

$$
\begin{aligned}
& \bar{m}_{30}=\sum_{k=1}^{N}\left(\cos \theta \cdot x_{k}+\sin \theta \cdot y_{k}\right)^{3} \\
& \bar{m}_{03}=\sum_{k=1}^{N}\left(-\sin \theta \cdot x_{k}+\cos \theta \cdot y_{k}\right)^{3},
\end{aligned}
$$

using the notation of a discrete shape representation consisting of $N$ feature points. An OII plot is generated by rotating the shape by an angle $\theta$ and computing the OII at each rotated position over the rotation range of $\theta \in[0,2 \pi)$. From (1) and (2), we deduce the following properties of the OII.

Property 1: The OII plot is periodic in $\theta$ with period $T=$ $\pi / 2$. That is $\mathrm{OII}_{\mathbf{X}_{\theta}}=\mathrm{OII}_{\mathbf{X}_{\left(\theta+\frac{\pi}{2}\right)}}$ for $\forall \theta \in[0,2 \pi)$.

Property 2: The OII plot has four peaks in the rotation range of $\theta \in[0,2 \pi)$.

Property 3: If two shapes differ from each other by a rotation angle of $\phi$, their OII plots are circularly shifted versions of each other by the same angular displacement $\phi$.

Property 4: If two shapes are reflected versions of each

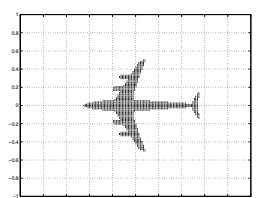

(a) Test Shape $\mathbf{X}$

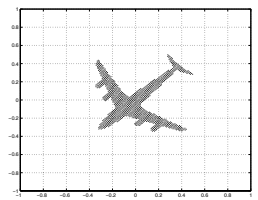

(c) Shape Rotated by $45^{\circ}$

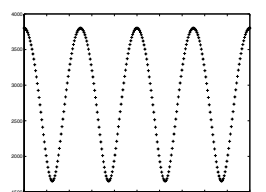

(b) OII plot of Test Shape

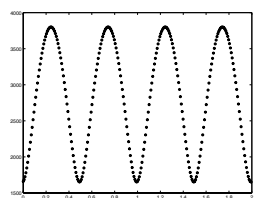

(d) OII plot of Rotated Shape
Fig. 1. Examples of OII

other about the $x$-axis, their OII plots are identical.

The second property requires a lengthy algebraic derivation to prove. After the derivation, we find that the OII plot is a cosinusoidal function of the rotation angle $\theta$ with a period $T=\pi / 2$. That is,

$$
\mathrm{OII}_{\mathbf{X}_{\theta}}=\sqrt{\frac{3}{4} \rho \cos (4 \theta-\lambda)+\tau}
$$

where

$$
\begin{aligned}
\rho & =\sqrt{\frac{\left(M_{1}-M_{3}\right)^{2}}{4}+M_{2}^{2}} \\
\lambda & =\arctan \left(\frac{2 M_{2}}{M_{1}-M_{3}}\right) \\
\tau & =\frac{5 M_{1}+3 M_{3}}{8}
\end{aligned}
$$

and

$$
\begin{aligned}
& M_{1}=m_{30}^{2}+m_{03}^{2} \\
& M_{2}=2\left(m_{21} m_{30}-m_{12} m_{03}\right) \\
& M_{3}=2\left(m_{12} m_{30}+m_{21} m_{03}\right)+3\left(m_{21}^{2}+m_{12}^{2}\right) .
\end{aligned}
$$

The OII plot represented by Equation (4) has four peaks over the range $\theta \in[0,2 \pi)$, which of course simply confirms Properties 1 and 2.

We illustrate the usage of the OII with simple examples. We first choose the test shape $\mathbf{X}$ to be the binary image of an airplane as shown in Fig. 1 (a). The test shape is rotated over the rotation range $\theta \in[0,2 \pi)$. At each rotated position, the OII value is computed and plotted. The OII plot that results after the full rotation is shown in Fig. 1 (b). As we expect, the plot is a cosinusoidal function of the rotation angle $\theta$ with a period $T=\pi / 2$. Fig. 1 (c) shows the same test shape rotated by an angle $\pi / 4 \mathrm{rad}$. The OII plot of this rotated shape is shown in Fig. 1 (d). Observe that this plot is circularly shifted by the angle $\pi / 4$ with respect to the OII plot shown in (b). 
The reorientation of a shape is achieved by first generating the OII plot. According to (4), there exist four peaks in the OII plot that represent four unique rotational orientations of the shape. We can bring any shape to one of its four unique orientations by bringing any peak in its OII plot to the origin $\theta=0$.

There are two major weaknesses in the method described above. The first limitation is that the method fails to resolve the reflectional ambiguity of the shapes as stated in Property 4 . Another very significant shortcoming is that the OII plot becomes flat over the entire rotation range $\theta \in[0,2 \pi)$ for certain shapes. When the OII plot is flat, there is no peak in the OII plot and the determination of the orientation is no longer possible. See the theorem below that states this fact.

Theorem 1 The OII plot of a shape is flat at zero if all of the third order central moments of the shape, $m_{30}, m_{03}, m_{21}$, and $m_{12}$, are zero.

\subsection{Variable-size Window OII}

In this section, we discuss the variable-size window OII ( $\Delta$ OII). The $\Delta$-OII is defined simply as the OII computed only from the portion of the shape falling within a $\frac{2 \pi}{\Delta}$-window where $\Delta=4 k$ for an integer $k$, i.e., we use windows of sizes $\pi / 2, \pi / 4, \pi / 8$, and so on. The $\frac{2 \pi}{\Delta}$-window is the region of the coordinate plane enclosed between the positive $x-$ axis and the straight line through the origin that makes an angle $\frac{2 \pi}{\Delta}$ rads with the $x$-axis. That is, the window contains all points $(x, y)$ where $x>0$ and $y \leq x \tan \left(\frac{2 \pi}{\Delta}\right)$.

The $\Delta$-OII of the shape $\mathbf{X}$ is defined as

$$
\Delta-\mathrm{OII}_{\mathbf{X}}=\sqrt{\mu_{x}^{2}+\mu_{y}^{2}}
$$

where

$$
\mu_{x}=\sum_{k \in R} x_{k}^{3} \quad \text { and } \quad \mu_{y}=\sum_{k \in R} y_{k}^{3},
$$

and $R$ is the region that contains the points $\left\{x_{k}, y_{k}:\left(x_{k}, y_{k}\right) \in\right.$ $\mathbf{X}, \forall x_{k}, y_{k} \in \frac{2 \pi}{\Delta}$-Window $\}$. The third order central moments $\mu_{x}$ and $\mu_{y}$ as computed for the $\Delta$-OII are never zero. This is because every $\frac{2 \pi}{\Delta}$-window is located within the first quadrant of the coordinate plane where the coordinate values of the points $\left(x_{k}, y_{k}\right)$ are greater than zero. The $\Delta$-OII plot, unlike the OII plot, never becomes flat at zero.

Two examples of the $\Delta$-OII plot are shown in Fig. 2. These are two of the shapes that cause failure on many existing methods. We observe that the $\Delta$-OII plot obtained from each of these shapes is (i) not flat and (ii) exhibits the rotational symmetry of the shape by its periodicity, i.e., the fold number of the shape is equal to the periodicity of the plot.

We briefly describe the algorithm for shape reorientation using the $\Delta$-OII. Readers are referred to [2] for details.

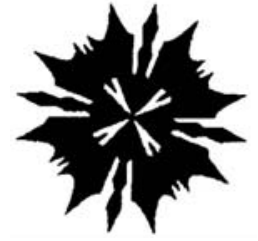

(a) Shape 1

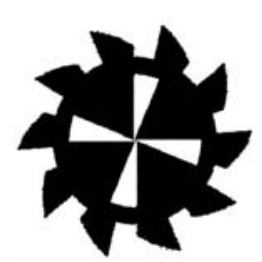

(c) Shape 2

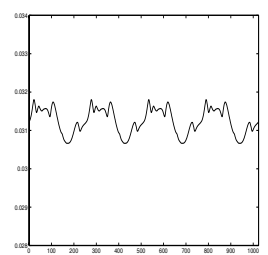

(b) $\Delta$-OII plot of (a)

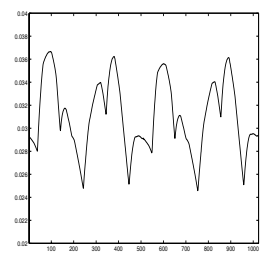

(d) $\Delta$-OII Plot of (c)
Fig. 2. Examples of $\Delta$-OII

The basic idea is as follows. Since the $\Delta$-OII plot is a function of the rotation angle over the full range $[0,2 \pi)$, if we locate a unique peak point of the plot and reorient the shape (i.e., rotate and reflect) until this unique point is brought to the origin at $\theta=0$, the shape can be uniquely reoriented. To achieve this, we first determine the fold number of the shape by plotting the $\Delta$-OII plot of the shape, generating an autocorrelation of the $\Delta$-OII plot, and counting the peaks with the magnitude of 1 in the autocorrelation plot. Then, we pick out the non-periodic portion corresponding to one period of the $\Delta$-OII plot and denote it as $\mathbf{L}$. We find the unique element in $\mathbf{L}$ by selecting the one with the largest value. If there is more than one such element, we resolve the tie by comparing the $\Delta$-OII values of their right-side and left-side neighbors in the $\Delta$-OII plot. Since $\mathbf{L}$ is non-periodic, we are guaranteed to resolve the tie and come up with a unique element in $\mathbf{L}$. However, any unexpectedly large error and noise in the $\Delta$-OII plot can disturb the correct result of the comparison process. A new method that works without these comparison steps will be beneficial. After the comparison steps are processed and we locate the unique element in $\mathbf{L}$, we perform an appropriate rotation and reflection to bring the shape to a unique orientation.

Every time we rotate the shape by an angle $\theta$, the $\Delta$-OII value at the rotated position is computed from a different portion of the shape that falls within the $\frac{2 \pi}{\Delta}$-window. This makes it difficult to derive the exact mathematical expression of the $\Delta$-OII as a function of the rotation angle $\theta$ as we did for the OII. We tested the $\Delta$-OII-based algorithm against an extensive database with two hundred symmetrical and non-symmetrical shapes [4] and verified that it correctly resolves all rotational and reflectional symmetries present. 


\subsection{Extended OII}

The $\Delta$-OII in (5) solves the problems exhibited by the OII in subsection 3.1. However, the $\Delta$-OII involves cumbersome and error-prone steps like comparing the $\Delta$-OII values of the given shape at different rotational positions. The extended OII $(X$-OII $)$ that we introduce now removes these steps.

So far, we have used only the third order central moments $m_{30}$ and $m_{03}$ of the shape in the OII and the $\Delta$-OII. For the normalized or compact shapes [5], the first and second order shape moments are normalized to $m_{10}=m_{01}=$ $0, m_{20}=m_{02}=1$, and $m_{11}=0$. Hence, when computing the OII in (1) for these compact shapes, the lowest order moments we are left to work with are the third order moments: $m_{21}, m_{12}, m_{30}$, and $m_{03}$. When applying the $\Delta$ OII, however, we are free to use moments of all orders, in particular, the lower order moments, $m_{10}, m_{01}, m_{20}, m_{02}$, $m_{11}, m_{21}$, and $m_{12}$, since these are now computed only within a $2 \pi / \Delta$ window, and so their values are not à priori fixed. This is what we do with the $X$-OII that we introduce next. The $X$-OII provides additional degrees of freedom in choosing the moments and is very useful in resolving, for example, reflection-symmetric shapes: either an individual moment can be used to generate the corresponding $X$-OII plot, or a few related moments can be combined to create a new measure of orientation as has been done in (1) and (5).

We give an example of processing reflection-symmetric shapes using the $X$-OII. When a shape is reflectionally symmetric, we have multiple peaks with the same $\Delta$-OII values within the non-periodic portion $\mathbf{L}$ of the $\Delta$-OII plot. We now explain how to avoid the comparison steps involved with the $\Delta$-OII. Consider the airplane shape in Fig. 3 (a) that is symmetric with respect to reflection about the $x$-axis. The $\Delta$-OII plot of this shape with a $\frac{\pi}{2}$-window, shown in (b), has two peaks due to the symmetry of the shape. To choose one unique element of the $\Delta$-OII plot, we need to compare the $\Delta$-OII values of the right-side and left-side neighbors of these two peaks. We employee the $X$-OII and generate two extra plots using $\mu_{30}$ and $\mu_{03}$ computed in the $\frac{\pi}{2}$-window, as shown in (c) and (d). This can avoid the cumbersome comparison steps: The peak in the $\mu_{30}$ plot corresponds to the right-side peak in the $\Delta$-OII plot, while the peak in the $\mu_{03}$ plot corresponds to the left-side peak. With this new index, $X$-OII, we can simply work with the $\mu_{30}$ plot, for example, and choose consistently the right-side peak in the $\Delta$-OII plot as the unique element.

\section{CONCLUSION}

The paper introduces moment-based measures of orientation, OII, $\Delta$-OII, and $X$-OII. We explore their properties and show that they remove the full orientation ambiguity including both rotation and reflection in all cases tested.

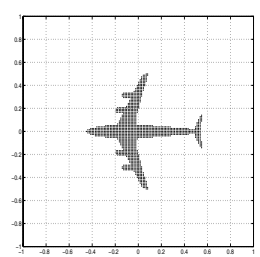

(a) Shape $\mathbf{X}$

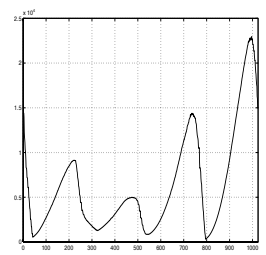

(c) $\mu_{30}$ Plot of (a)

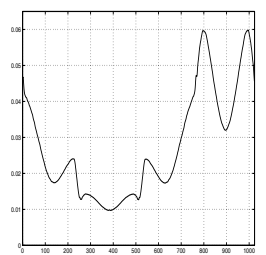

(b) $\Delta$-OII plot of (a)

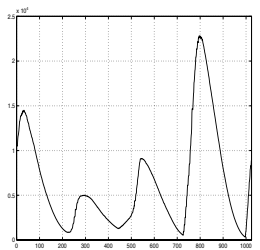

(d) $\mu_{03}$ Plot of (a)
Fig. 3. Examples of $X$-OII

These OII's use low order moments and are robust to errors and noise. The extended OII ( $X$-OII $)$ is free of the error-prone steps of the other two OII measures and works especially better on the shapes with reflectional symmetry.

\section{REFERENCES}

[1] V. H. S. Ha and J. M. F. Moura, "Intrinsic shape," in The 36th Asilomar Conference on Signals, Systems and Computers, Monterey, CA, November 2002, vol. 1, pp. 139-143.

[2] V. H. S. Ha and J. M. F. Moura, "Efficient 2D shape orientation," in IEEE International Conference on Image Processing, 2003, vol. 1, pp. $225-228$.

[3] D. Shen and H. Ip, "General affine invariant image normalization," IEEE Transactions on Pattern Analysis and Machine Intelligence, vol. 19, no. 5, pp. 431-440, May 1997.

[4] D. Shen and H. H. S. Ip, "Symmetry detection by generalized complex (GC) moments: A close-form solution," IEEE Transactions on Pattern Analysis and Machine Intelligence, vol. 21, no. 5, pp. 466-476, 1999.

[5] J. G. Leu, "Shape normalization through compacting," Pattern Recognition Letters, vol. 10, pp. 243-250, October 1989. 